\title{
University Students' Perception of Their Own Academic Achievement Motivation: A Field Study
}

\author{
Fares Hilmi ${ }^{1}$ \\ ${ }^{1}$ Department of Psychology, Faculty of Arts, University of Jordan, Amman, Jordan \\ Correspondence: Fares Hilmi, Department of Psychology, Faculty of Arts, University of Jordan, Pobox 13528, \\ Amman 11942, Jordan. E-mail: Faresjabr@yahoo.co.uk
}

Received: February 3, 2013

Accepted: March 19, 2013 Online Published: April 18, 2013

doi:10.5539/ijbm.v8n10p67

URL: http://dx.doi.org/10.5539/ijbm.v8n10p67

\begin{abstract}
The aim of the present two-stage study was two-fold: first, to identify motivational themes thought of by university students themselves as to what causes their very high motivational learning states, and also their very low motivational states; and second, to determine the underlying dimensions affecting both of these states.

Undergraduate students studying at a major government university in Jordan were used in this study. All of the subjects were from different colleges and were enrolled in an elective university course. 534 subjects were used in stage one of the study, and 304 other students were used in stage two.

A modified version of the Critical Incidents Technique was employed to elicit various themes considered by university students as causes of states of very high and very low motivation to study. It was assumed that all that university students think of as learning-encouraging or learning-discouraging represents very high and very low learning motivational themes. Content analysis quantification was carried out in order to determine a university students' motivational themes list.
\end{abstract}

Using the Principal Component Analysis statistical technique to cluster-analyze the 14 very high motivational state themes resulted in four motivational dimensions. These dimensions are as follows: 1) self-determination, 2) positive academic attitudes, 3) need for social respect, 4) self-monitoring.

Cluster analysis of the 10 very low motivational state themes produced three dimensions, which are as follows: 1) negative personal feelings and circumstances, 2) learned helplessness, 3) negative self-monitoring.

Keywords: academic achievement, motivation, university students, critical incident

\section{Introduction}

The purpose of this study was to investigate what motivates university students in Jordan to study very hard indeed, or conversely not to study hard at all, in their efforts to master those learning objectives specified by their university educational plan.

Though psychologists have studied motivation in general intensively, Jacobs \& Newstead (2000) stated that "little is known about what motivates university students: why they embark on their studies in the first place". In addition, Lavery (1999) noticed that very little research focused on the academic motivation of students in a multicultural university setting. Moreover, a major part of the present body of knowledge concerning university students' motivation dealt with this important issue in a fragmented way: researchers studied only certain aspects of this complex situation.

For example, Jacobs et al. (2000) employed the concepts of knowledge, skills, and experience (developed by Radford and Holdstick, 1993) to study university students' motivational preferences. Their study found that there was a specific structure to the topics that motivate students, that their motivation was affected by their year of study at university, and that female students were more highly motivated than males. Biggs (1987) used different categories to portray students' motivational preferences: surface, deep, and achieving. Entwistle and Entwistle (1991) showed that final year university students were more interested in reproducing motivation orientation rather than meaning orientation.

Other studies detailed further results of interest. For example, Walters (1998) found that university students monitor and regulate their own motivation in order to complete their academic tasks. Tang (1990) found that 
Taiwanese students with a low work ethic exert more effort when they are challenged. Watkins and Hattie (1981) found that the students who were most in need of counseling in study methods were young students, male students, and students enrolled in science-based faculties. A different line of research attempted to understand university students' academic motivation by applying the dichotomy of internal versus external motivational factors. Other terms were used to depict more or less the same dichotomy, such as intrinsic versus extrinsic, and autonomous behaviour versus exonomous behaviour. Recently, the term "self-determination" has been borrowed from the political science domain and used eloquently by Deci, Connell \& Ryan (1989) to represent the same paradigm.

Most recently, however, this dichotomous approach-though well-established and very useful - has been criticized in an attempt to develop a multi-dimensional perspective for understanding learning situation outcomes, in particular those situations where students cannot always choose the learning tasks they like best. (Often students have to learn certain tasks which they do not like before they are allowed to learn what they do enjoy.) University learning situations are very complex and may, therefore, not be well understood with a one-dimensional approach. Hwang (2002), for example, found that students quoted positive outcomes and also learning itself as reasons for studying. This can be interpreted as successful cohabitation between intrinsic and extrinsic motivational factors. In fact it has been found repeatedly that intrinsic and extrinsic motivational factors are not mutually exclusive and may indeed coexist with various types of motivational factors (Dawson \& Mclnerney, 1997, 2001; VanEtten, 1997; Hwang, 2000).

The present study aimed, therefore, to take this broader multi-dimensional approach into a new research ground, i.e. into a different culture which had not yet been studied using this particular approach. Indeed, compared with the overall number of studies conducted in North America and Europe, very few motivational studies per se have been conducted in Middle Eastern countries at all. It is hoped, then, that this study may add to and enrich that small body of existing research relating to Middle Eastern culture and thereby widen the scope for cross-cultural studies of this nature, whilst at the same time revealing results both relevant and useful to the process of renovation and restructuring currently taking place in Jordan, one Middle Eastern country. The urgent need for such change in Jordan was illustrated very convincingly in the United Nations Development Plan (UNDP) Human Resources Development Report, 2003. One of the change targets for the Middle Eastern countries (Jordan included) is the quality standard of university graduates. In this regard, the motivational aspects of university students' learning situations are very relevant indeed, as shown very frequently in psychological learning research. Though the motivation component of learning has been somewhat neglected so far in favor of the non-student aspects of the learning situation, such as instructors, classrooms, regulations and learning technology, in Jordan a very small fraction (one-tenth of a percentage point) of the score in his or her Secondary Education School Leaving Certificate ("Tawjihi") examinations may be all it takes to consign a student to a college or a specialty that $\mathrm{s} / \mathrm{he}$ does not like and does not wish to pursue as a career. Moreover the individual student may not be able to do anything about this and thus has to live with the consequences.

It is clear that motivational issues are culturally loaded and are affected by both the political and economic state of affairs in a country. The social values system relating to job preference and job availability cannot be discounted. In a pre-industrialized country such as Jordan the processes of employee selection and employee annual performance appraisal are not well-developed. Therefore, a university degree will most probably be a key factor in gaining employment and indeed remaining in one of the scarce jobs in the country. The official unemployment rate is approximately $15 \%$, and the unofficial rate as determined by the University of Jordan Strategic Studies Center is $21 \%$, hence the importance of a university degree cannot be over exaggerated. Such a situation does not exist in industrialized countries, where an employee's skills and productivity, in most cases, are the basis for hiring and firing.

Entwistle et al. (1971) stated that motivational scales developed and used in the United States are not altogether suitable for use in Britain. Obviously the cultural similarities between the USA and Britain are far greater than those between the USA and Middle Eastern Arabic-speaking countries. With this in mind, the present study needed to be designed for as well as conducted in a university in Jordan in order to uncover those motivational components specific to university students in this particular country and culture.

It was decided that this study would not merely try to validate one of the famous motivational theories, or one of the available motivational scales developed to assess specific motivational concepts. Such an approach would be very liable to miss some important motivational themes applicable to the people of the culture under investigation (Tannenbaum, 1980; Barret \& Bass, 1976). To achieve the aim of the present study, the university learning process needed to be considered as a complex situation and looked at in a holistic manner. 
This study also adopt the view of certain motivation theorists who looks upon the individual (the university student in the present case) as a rational decision-maker, who consciously and cognitively judges and evaluates the situation, and assesses his or her personal benefits and costs, before taking action (Adams, 1965; Vroom, 1964). In this study, 'very high academic motivational themes' refers to any factors which encourage students to study very hard. In this state of high motivation (studying very hard), the individual student will be wholly engaged with the learning objective and dedicate whatever it takes in effort and time to master the learning task. Conversely, 'very low academic motivational themes' refers to any factors which encourage students not to study very hard. These create a state of very low academic motivation where the individual student is not wholly engaged with the learning objective. He/she will be distracted from the learning task and will devote very little effort or time to the process of mastering the learning task.

Such academic motivational themes can be personality characteristics, conditions, events, feelings, attitudes, environmental situations, family circumstances, significant others, and university learning objectives and regulations.

Finally, the present investigation was guided by the following questions: What are the themes that university students report as the direct or indirect causes which encourage them to work extremely hard in terms of time and effort in order to participate in the learning process and master the educational tasks required of them?

What are the themes that university students report as the direct or indirect causes which greatly discourage them and significantly reduce their willingness to participate in the learning process, hence decreasing significantly the effort they assign to master the educational tasks required?

What are the underlying dimensions (groups of themes) that encourage students to be involved in the learning process and learn studiously; and conversely, what are the underlying dimensions (groups of themes) that discourage students from studying very hard and result in them devoting very little time and effort to mastering the learning task?

The above-mentioned guiding questions were answered in two stages: Stage 1 answerd the first two questions, i.e. identifying the university students' academic achievement motivational themes for studying very hard (high motivation), and the themes for studying little (low motivation); Stage 2 will focus on determining the underlying motivational dimensions embedded in the relationships among the motivational themes identified in Stage 1.

\section{Stage 1: University Students' Academic Motivational Themes}

\subsection{Method}

\subsubsection{Participants}

A total of 528 undergraduate students in a major state university in Amman, the capital of Jordan, participated in the first stage of this study. Students were enrolled in various colleges and were attending five social psychology classes. This sampling method can be termed 'opportunistic' or 'convenient' (Clark-Carter, 1997). The participants' mean age was $20(\mathrm{sd}=4$ years). Three hundred and ten were females and 220 were males. 96 students were from year 1; 189 students from year 2; 138 from year 3; and 98 from the final year. Seven students failed to identify the year of their study in the university.

\subsubsection{Setting, Materials and Procedure}

In the context of a classroom setting and time, the students enrolled in these five social psychology classes (an elective course for university students) were asked to participate voluntarily in an academic study in the area of social psychology. They were told that there would be no wrong or right answers, that what was needed was simply to write down their thinking honestly and accurately, and that whatever they wrote would be strictly confidential. The administration of the open-ended survey lasted 15 to 17 minutes.

Participants were asked to complete a modified version of the Critical Incidents Technique (Flanagan, 1954; Herzberg et al., 1959), the goal being to elicit university students' perceptions, i.e. ideas, thoughts, views, opinions, beliefs, and feelings, related to those occasions when they studied very hard (assumed to be high motivation), and to those occasions when they felt that they did not study hard (assumed to be low motivation). The causes could be anything the student thought of as being a direct or indirect cause of the occurrence of the state of high/low motivation.

In order to generate responses representing students' perceptions of causes that encouraged them to study exceptionally hard or to study exceptionally little, two open-ended questions were presented. The first question asked students to remember occasions when they participated whole-heartedly in the learning process to achieve 
the educational objectives, occasions when they invested an exceptional amount of effort and time to master the learning tasks. They were asked to write a brief description of such occasions. They were then asked to write down in the blank spaces three different direct or indirect reasons for studying very hard. This question represented the state of high motivation. The second question asked students to remember occasions when they were exceptionally uninvolved in the learning process, when they invested exceptionally little effort and time to attain the learning objectives. They were then asked to briefly describe such occasions. Again they were asked to write in the blank spaces three different direct or indirect reasons for the occurrence of such a low motivation state. (24 students failed to answer this second question.)

Finally, the students were asked to give some demographic data, their age, year of study, total grade average, and specialty or college.

\subsubsection{Pre-Testing Procedure}

Before the actual data collection began, the survey instrument was pretested to ensure that students would understand the questions accurately and that the elicited responses would be in line with those expected. The survey, together with the operational definition, was therefore passed to a group of five psychology lecturers for comments. A few changes were made to the wording of certain sentences. The next step was administering the survey to a class of 27 psychology students at second and third year levels. The analysis of the students' responses revealed that all were of the expected type. Responses to the first question were all related to a high motivation state, and the second question elicited responses related to a low motivation state. Therefore no further changes were made in the layout or the wording of the survey.

\subsection{Content Analysis}

After the data collection, participants' responses to the first and second questions were content-analysed separately, yet in a similar manner. The quantification of responses (sampling and finding themes) followed the steps and recommendations of Sommer \& Sommer (1986). A random sample of 100 completed questionnaires was selected to be utilised to establish themes reflecting the high motivation states of university students, as well as themes reflecting their low motivation states. The author of the present study trained two postgraduate students hoping to continue their studies at $\mathrm{PhD}$ level on content analysis steps and procedures. All responses in the random sample were then analysed and coded for content and meaning by each of the two coders independently. After this, the lists created by the two coders were used to create a single list for the high motivation themes, and another single list for the low motivation themes, a theme being an idea representing whatever encouraged or discouraged involvement in the learning process. Any disagreement was resolved through discussion, under the supervision of the author.

The resultant list of motivational themes was used to analyse participants' responses in the remainder of the survey questionnaires. The inter-rater reliability coefficient values for high and low motivation themes were $\mathrm{K}=$ 0.82 , and $\mathrm{K}=0.80$, respectively. This indicates a high level of agreement between both coders.

\subsection{Content Analysis Results}

Each of the two coders categorised the remainder of the elicited responses. The results of the content analysis are presented in Table 1, which details high and low motivational themes and their meanings, together with the frequency and corresponding percentage of subjects mentioning each theme.

\subsubsection{Students' High Motivational Themes (Students' Motivators)}

Perhaps most striking is the fact that half of the students (49.6\%) who participated in the study mentioned the goal of achieving high performance grades. This theme was expressed in many different ways, but centered about the hope of getting excellent scores in exams, to keep the total average score as high as before. It seems that this is the most important single driving force behind students' academic activities while at university.

The second most frequently-mentioned theme is the students' love for achieving distinction and a positive response to challenge and competition. $29 \%$ of the subjects mentioned that a major reason for studying hard is to face up to the difficulties and confront situations and come out as winners.

The third most frequently-mentioned motivating theme (24.1\%) is a positive attitude towards science and knowledge in general: a kind of curiosity, a need to know how things work or to understand things. A very frequent response was "/like scientific knowledge". This inner willingness to acquire scientific knowledge is a significant component of the subject's character.

Three further themes, all appearing to embrace the concept of satisfaction, were mentioned by very similar percentages of students: pleasing one's parents (21\%); being accepted in the speciality of choice (20\%); and the 
feeling of satisfaction that one gets from studying hard and achieving hoped-for results (19\%). The strong family ties inherent in Jordanian culture are clearly apparent here - students want to make their parents proud of them, to give their parents satisfaction. On the theme of specialty, some students wrote "to be accepted in the department I like", "to be admitted into the specialty 1 really want," "to be accepted into the specialty I long for." As for the theme of satisfaction resulting from studying hard, some students wrote "self satisfaction," "to be happy after doing my duty," and "to satisfy my conscience."

It is interesting to notice also that three motivating themes all appearing to embrace the concept of possible failure were mentioned by exactly the same percentages of students (15\%). Fear of failing and fear of the exam motivates students to study harder. Also, whenever they find that the learning task is more difficult, they are motivated to devote more effort and spend longer periods of time studying if necessary. Gaining the respect of others, friends, and significant others in society at large was mentioned, referring to culture and society at large, by saying that "people do not like lazy individuals." "people like successful individuals'7. Other examples of phrases used were "to keep my standing in the eyes of my friends." "to be liked by my friends." "to gain the respect of others." "to have a high standing social position."

The remainder of the motivating themes mentioned by students include to secure a job in the future (13.3\%). There is no mention of a specific job or career aspiration, it is merely the ensuring of a job - some kind of guarantee or insurance for the future. The speciality factor was previously mentioned as a reason for studying hard, but students also mentioned their preference for the course materials or the learning tasks as an encouragement to master the learning task (11.6\%). A similar percentage of students mentioned the theme of self-realisation or self-actualisation (11\%). Phrases used in this category were "to prove my self," and "to achieve my ambitions." The theme of graduation or quick graduation (10.1\%) was mentioned as a goal in itself, as if somebody was waiting for him/her, or as if there was a timetable and $\mathrm{s} / \mathrm{he}$ has to meet that target date.

The last theme with a percentage of note was mentioned by a small number of students (siuprisingly only 7.4\%) and this is the influence of lecturers. This is surprising since university administrative bodies spend considerable time and resources on improving the pedagogical effectiveness of the academic staff. Examples of the expressions used in this regard are as follows: "to satisfy my teacher." "lecturer's style." "lecturer's positive influence."

The final three motivating themes were mentioned by a very small percentage of subjects. Suitable personal circumstances do encourage students to participate whole-heartedly in learning situations and to try to master learning tasks, but this motivator was mentioned only by 11 subjects $(2.1 \%)$. The religion factor was mentioned by an even smaller number of subjects - $9(1.7 \%)$. Some responses were: "to be a good Muslim." "obeying God." "behaving according to the teachings of Islam."

Lastly, task easiness was mentioned by only five subjects (1\%).

Those students work hard if the course material is easy. These final three motivators with percentages less than $3.5 \%$ will not be carried forward to the next stage, since such percentages are very small indeed, and for the sake of brevity.

Table 1. Motivational themes identified through content analysis of open-ended questions

\begin{tabular}{|c|c|c|c|}
\hline & Themes & Frequency & Percentage \\
\hline & High Motivational Themes (Motivators) & & \\
\hline 1 & $\begin{array}{l}\text { High Scores. To achieve scores in exams, to achieve high marks, for the sake } \\
\text { of a very good score, to achieve excellent marks }\end{array}$ & 262 & 49.6 \\
\hline 2 & $\begin{array}{l}\text { Love of challenge and winning in competition, to compete with other students, } \\
\text { I like to be the first among my group and colleagues }\end{array}$ & 153 & 29 \\
\hline 3 & $\begin{array}{l}\text { Love of science in general, I like to understand how things work, I like to } \\
\text { know more about things, I like scientific knowledge, it is curiosity }\end{array}$ & 127 & 24.1 \\
\hline 4 & To please my parents, to satisfy parents, to make my parents happy & 113 & 21.4 \\
\hline 5 & $\begin{array}{l}\text { Specialty I Like. To be admitted in the department I like, the specialty I long } \\
\text { for, Achieve marks to get into the specialty I really want, to get a grant }\end{array}$ & 109 & 20.6 \\
\hline 6 & $\begin{array}{l}\text { Feeling of Happiness, feelings of self satisfaction, in order not to be blamed, to } \\
\text { satisfy my conscience }\end{array}$ & 101 & 19.13 \\
\hline 7 & Failure avoidance, fear of failing, fear of exam & 83 & 15.7 \\
\hline
\end{tabular}


8 Task difficulty, course material is very difficult, specialty is demanding

80

To get the respect of others, to get the admiration of my friend, to be liked by

9 people, to keep my standing in the eyes of my friends, to be liked by my friends, society do not like lazy people, to get a prestigious position

10 To secure a job in the future, to have a better future, to get a job very quickly, some kind of a guarantee in the future, to have a better future

Specialty courses and subjects. I like the courses, I study in the specialty I

11 love, the courses I study are very interesting, studying the courses in my specialty are very joyful, it is fun to study the courses of my specialty

Ambition and self-actualization. To achieve my ambitions, to realize my hopes, to prove my-self, to realize my aspirations

13 Quick Graduation, I am looking forward to graduation, I hope for an early graduation, to pass quickly

4 Lecturers positive influence, to satisfy my teacher, lecturer's style, my teacher encouragement

15 Suitable personal circumstances

16 Religion teachings, in order to be a good Moslem, obeying God

17 Task easiness, course material is easy

18 Handwriting is not clear

20 Cannot be classified

\section{Low Motivational Themes (Demotivors)}

Negative Psychological states such as boredom, frustration, laziness, feelings of hopelessness, I feel I am lost. I am always tense

Negative personal circumstances, do not have enough time, unhelpful environment, feelings of being tired and exhausted

Negative feeling towards specialty, I do not like studying this specialty, I hate

3 this subject, I like to study another specialty, I do not care for this course, a boring course

4 Task easiness, very easy course material, I know the material, there is nothing scientific new in the course worth studying, do not need to master

Task difficulty, I do not understand the course material, very difficult course subject, I cannot absorb what is taught

Cannot concentrate, always I want to do other things, the future, thinking of our state of living, thinking of the people that I love

No positive rewards, low scores will never change whether worked hard or 65

11 Moving to another place, I am waiting to go to another university, going to another country, I hope to go to another university

12 Lack of exams, I study just for exams

13 Friends negative influence, busy with friends Negative feelings towards the lecturer, I hate the lecturer, a difficult lecturer, lecturing style, lecturer do not encourage us to study the course material University regulations and system, late lectures, bad transport, exam times

15 are badly organized, plenty of credit hours, one exam and different teaching styles, exam scores will not be affected

\section{.2}


16 Feelings towards exams. Exams are difficult, difficult exam questions, fear of exam, task materials are huge and beyond mv ability

8

No way to continue in the university, do not have enough abilities to stay in the university and study

IS Not clear

19 Cannot be coded

20 Repetition

Total sample subjects 528
22

61

\section{5}

0.6

5.3

4.2

11.6

\subsubsection{Students' Low Motivational Themes (Demotivators)}

The reasons given for studying very little were content-analysed into 17 demotivational (discouraging) themes, detailed in Table 1 in descending order of frequency. The most dominant theme, which was mentioned by $41 \%$ of the respondents, is negative psychological states. Among responses categorised under this theme are those relating to frustration, being fed up with studying, boredom, feelings of hopelessness and helplessness, and being in a state of despair. The second most frequently-mentioned theme is negative personal circumstances (27.7\%), including having insufficient time for studying, an unhelpful environment, and experiencing feelings of tiredness and exhaustion. The third most frequently-mentioned theme is negative feelings towards one's speciality (19.7\%). Students expressed in the strongest possible terms their dislike of their specialty: ' 7 do not like studying this specialty", "do not care for this specialty, '7 like to study another specialty." "I hate my specialty." It is not surprising that such dislike of the specialty created a lack of willingness to learn or master a set task.

Concerning the learning task level of difficulty, if the task was easy and they were familiar with it, $19.7 \%$ of subjects responded that they would not try very hard to learn it. Conversely, if the task was judged by the student to be very difficult, then $14.8 \%$ of students would again not try to master it. Some students responded that if the subject is very difficult, they cannot understand it, and cannot absorb the course material. An equal percentage of subjects (14.8\%) mentioned that they could not concentrate on the learning task, often stating that they were thinking of other things, or the people that they care for and love.

Approximately the same percentage of subjects (12\%) mentioned negative outcomes as mentioned negative feelings towards the lecturer as a very demotivating factors. If their experience showed that "no positive results". "no rewards" or "no positive feedback." "no good scores" can be expected from the learning activity, then the subjects would not try again to master that particular educational task. Related to this absence of positive feedback, 64 subjects mentioned negative attitudes and feelings towards their lecturers: "I hate my lecturer," a difficult lecturer," "lecturer style," and "lecturer does not enourage us to study the course materials."

Related to this a total of $11 \%$ of subjects mentioned wrong information and wrong beliefs they had about the university system of learning as reasons for not studying as hard as they should be: "I thought succeeding in the university can be achieved without great effort." "did not know the importance of the average grade." "I did not realize that achieving high marks is very important in university regulations."

Negative family circumstances were mentioned by $10.2 \%$ of students as affecting their learning. They cited states such as "high tension inside the family', "lots of visits." "high family expectations of me." "worried about the health of..." one parent or both, "family duties." Obviously some students' general circumstances and surroundings were not conducive to learning and did not allow students sufficient time for the learning process.

Just as the lack of accurate information was cited as a reason for not studying hard, so too was the lack of adjustment to the new situation (life at university): "Do not know the best way to study in the university." "very big change from school to university." "could not cope with the university life." These are some examples of responses given by $5.9 \%$ of the students and categorised in this theme of demotivators.

The remaining study-discouraging themes are detailed in Table 1, but all were mentioned by less than $3.5 \%$ of subjects. One of these themes shows the subjects in a state of transition, waiting to go to a different university or be accepted in another specialty, or even moving to a different country. This will inevitably affect the student's involvement in the learning process. Lack of exams is another theme: some university students study only when they have to pass an exam, therefore studying only during exam times. Friends may also have a negative influence -and being with one's friends may not leave the student with sufficient time for studying. University transport, exam times organisation, a heavy credit hour load, all can negatively affect some students such that they cannot manage their time in the university efficiently. A further demotivating theme, mentioned by $1.5 \%$ of subjects, is exam phobia: "fear of exams," "difficult exam questions," The final theme affecting students' 
participation relates to their hopes of continuing in the university. (This state was mentioned by just three subjects.) If the student loses hope, he or she will not participate in the learning process. Hope can be lost for various reasons, one of which may be that if in his or her experience no rewards come, then it is perhaps to be expected that students will stop trying to learn.

To summarise, the purpose of Stage 1 of the present investigation was to identify themes perceived by university students themselves as being direct or indirect causes of their states of high and low academic motivation. This purpose was achieved and the motivational themes are presented in Table 1. As previously stated, only those motivators and demotivators mentioned by more than $3.5 \%$ of subjects were carried forward to Stage 2 of the investigation for the purpose of factor analysis to identify the students' underlying academic motivational dimensions.

\section{Stage 2}

\subsection{Motivational Themes Clustering}

The aim of the second stage of this study was to establish those basic dimensions underlying students' motivational themes as determined by the content analysis completed in Stage 1. This aim could be achieved through grouping these themes in several different ways. Experts rely upon their own understanding of the motivational themes. They may employ the principal of themes' meaning similarity to categorise the different phrases into a certain category or under a certain heading. Another approach is to categorise the different themes employing the existing categories of a certain theory. Different experts may choose different theories. The choice range is very wide indeed, from need theories, to rational theories, to task characteristics models, to goal setting and reinforcement theory. However such approaches to establishing the basic dimensions underlying this particular study's students' motivational factors most probably, however, would reflect a cultural bias, since these approaches originated in foreign (mainly western) countries. It was therefore decided to generate the basic dimensions underlying students' motivational factors through applying a statistical technique to students' responses on a five-point Likert-style scale based on the results of the content analysis.

\subsection{Method}

Questionnaire, procedure, participants and data gathering

All of the high and low motivational themes in Table 1 with percentages of mention higher than $3.5 \%$ were used to construct a Students' Academic Motivation Questioimaire. The total number of motivational (encouraging) themes representing high motivation was 14 , and the total number of demotivational (discouraging) themes representing low motivation was 10 . The phrases which were used to construct the questionnaire were the most frequently mentioned phrases used by the students themselves in each theme.

The scoring was on the basis of a five-point Likert-type scale, as follows: 1-not sure; 2-little importance; 3-moderate importance; 4- important; 5-very important.

In the questionnaire, for the high motivational themes students were asked to indicate, on the basis of their own experience, to what extent each theme was important for their own exceptionally great involvement in the learning process when they studied very hard, and for the low motivational themes, they were asked to indicate to what extent each theme discouraged them personally from studying hard and therefore could account for situations in which their own involvement in the learning process was minimal.

After thus constructing the questionnaire, it was passed to a group of nine psychologists for language and meaning review. Very few changes were suggested. The revised version was then administered to a small class of psychology students (25 students at final year level). The students' responses were in line with those expected, and there did not appear to be difficulties in understanding the questions or in how to respond accurately. The questionnaire was then administered to five classes of social psychology students (305 students) a year later, in the same university where the first stage of this study had been completed. This was to ensure that new students had enrolled in those classes. Administration of the questionnaire took place in a classroom setting, in the final 20 minutes of a lecture: students were given the choice of leaving the classroom or participating in answering the questionnaire. Very few students left. On average, students required 12 minutes to read the instructions and answer the questionnaire. After collecting the questionnaires and examining the students' responses, 21 questionnaires were discarded for lack of demographic information, or for the presence of an indication that it was not answered in a serious or honest manner.

\subsection{Demographic Data}

The subjects' mean age was 20.3 years $(\mathrm{sd}=1.7)$. There were 70 males $(24.6 \%)$ and 214 females $(75.4 \%)$. There 
were 93 subjects in year 2(32.7\%), 150 subjects in year 3(52.8\%), and 41 subjects in year 4(14.5\%).

3.4 Statistical Analysis

Cronbach's alpha was calculated to establish the internal consistency reliability of the questionnaire. For the high motivational themes an alpha of 0.74 was obtained, and for the low motivational themes an almost identical alpha of 0.73 was obtained. These results indicate that the questionnaire has an acceptable level of internal consistency. A Principal Component Analysis with varimax rotation was performed on the high and low motivational themes to generate the underlying dimensions. An eigen value of $>1$ was chosen to determine the number of dimensions to be extracted from the data. Factor coefficient loadings had to be greater than 0.4 to be included in the principal component factors.

\subsection{Results}

\subsubsection{Statistical Analysis Results}

To cluster-analyse the 14 high motivational themes and the 10 low motivational themes, Principal Component Analysis (PCA) was used for both parts of the questionnaire. The 14 high motivational themes produced 4 main high motivational dimensions which accounted

for $51.6 \%$ of the variance in the data, with eigen values of 1 or more. The first dimension with loadings above 0.529 on themes $\mathrm{H} 3, \mathrm{HI}, \mathrm{H} 10, \mathrm{H} 2, \mathrm{H} 9$, and $\mathrm{H} 4$ accounted for $24.87 \%$ of the data variance. The second main high motivational dimension with loadings above 0.558 on themes H12, H5, and H6 accounted for $10.92 \%$ of the data variance. The third main high motivational dimension with loadings above 0.495 on themes $\mathrm{H} 9, \mathrm{H} 8$, and $\mathrm{H} 7$ accounted for $8.04 \%$ of the variance. The fourth and final main high motivational dimension with loadings above 0.465 on themes $\mathrm{H} 4$ and $\mathrm{H} 14$ accounted for $7.79 \%$ of the variance. Theme H9 is part of dimensions 1 and 3, and theme $\mathrm{H} 4$ is to be found in dimensions 1 and 4.

Table 2. Results of the SPSS principal components analysis of the items of the motivational themes questionnaire

\begin{tabular}{|c|c|c|c|c|c|}
\hline & High Motivational Themes (Motivators) & 1 & 2 & 3 & 4 \\
\hline 1 & My wish to achieve high average scores & $.632 *$ & .006 & .050 & .081 \\
\hline 2 & Feelings of satisfaction and happiness after doing my duties & $.574 *$ & .127 & .023 & .073 \\
\hline 3 & To get average score high enough to be accepted in the specialty I like & $.680^{*}$ & .186 & -.104 & .075 \\
\hline 4 & To achieve and realize my ambitions and hopes from university studying & $.529 *$ & .169 & -.105 & .465 \\
\hline 5 & Because I love my specialty courses and subjects & .338 & $.708^{*}$ & -.147 & -.109 \\
\hline 6 & Because I love science and knowledge in general & .344 & $.558^{*}$ & .266 & -.012 \\
\hline 7 & My wish to graduate very quickly from the university & -.165 & .188 & $.698^{*}$ & .022 \\
\hline 8 & To earn the respect and admiration of my friends and others in general & .257 & -.154 & $.700^{*}$ & .196 \\
\hline 9 & Because I love my parents and to please them & $.562 *$ & -.147 & .495 & -.062 \\
\hline 10 & I love competition and challenge to achieve excellence and distinction & $.585^{*}$ & .142 & .334 & -.039 \\
\hline 11 & To make sure I will get a job in the future & $.393 *$ & .141 & .198 & .275 \\
\hline 12 & Lecturers style in teaching and his positive influence & -.085 & $.769^{*}$ & .004 & .176 \\
\hline 13 & Challenging tasks and demanding specialty makes me work harder & .313 & .279 & .162 & $.348^{*}$ \\
\hline \multirow[t]{2}{*}{14} & Fear of failure and failure avoidance & .000 & -.050 & .088 & $.885^{*}$ \\
\hline & Low Motivational Themes (Demotivators) & 1 & 2 & 3 & \\
\hline 1 & Feelings of frustration, boredom and hopelessness & $.735^{*}$ & .116 & .119 & \\
\hline 2 & Cannot concentrate, always I have to do other things & $.753 *$ & -.076 & .161 & \\
\hline 3 & Negative personal circumstances, do not have enough time & $.760^{*}$ & .283 & -.104 & \\
\hline 4 & Task difficulty, and inability to understand the course work & $.534 *$ & .148 & .259 & \\
\hline 5 & A belief that university does not require lot effort to succeed & .207 & -.027 & $.608^{*}$ & \\
\hline 6 & I hate my specialty and I do not like it & .075 & $.736^{*}$ & .155 & \\
\hline 7 & Task easiness, the very easy course work does not warrant any effort & -.034 & .114 & $.761^{*}$ & \\
\hline 8 & Negative family state and circumstances & .440 & $.650^{*}$ & -.112 & \\
\hline 9 & No hope to improve my average score, i.e., there is no hope positive rewards & .296 & $.408^{*}$ & .390 & \\
\hline 10 & $\begin{array}{l}\text { Lack of adjustment with the university environment and life could not cope } \\
\text { with university life }\end{array}$ & -.003 & $.712 *$ & .034 & \\
\hline
\end{tabular}


Note: an Eigen value $>1$ was chosen to determine the number of dimensions to be extracted from the data, and Factor coefficient loadings had to be greater than 0.4 to be included in the principal component factors. Four components extracted from the high motivational themes and three components from the low motivational themes.

Similarly, a Principal Component Analysis (PCA) on the 10 low motivational themes produced three main low motivational (demotivational) dimensions which accounted for $53.31 \%$ of the variance in the data, with eigen values above 1 . The first main demotivational dimension with loadings above 0.44 included themes L3, L2, LI, L4 and L8, and accounted for $30.2 \%$ of the data variance. The second main demotivational dimension with loadings above 0.408 included themes L6, L10, L8 and L9, and accounted for 12.21\%) of the data variance. The third main demotivational dimension with loadings above 0.61 included themes L7 and L5, and accounted for $10.9 \%$ of the variance of the data. Theme L8 was included in both main dimensions 1 and 2, but with a higher loading in dimension L2.

\section{General Discussion}

The present study aimed to determine the causes, conditions and events that encourage university students in Jordan to study exceptionally hard (high motivation), and those that greatly discourage them from studying (low motivation). After content-analysing those causes into encouraging (motivating) and discouraging (demotivating) themes, a further aim was to factor-analyse them into groupings representing university students' underlying motivational dimensions. These high and low motivational dimensions will now be discussed in turn.

\subsection{University Students' High Motivation Dimensions (Motivators)}

A Principal Component Analysis was carried out on the 14 study-encouraging themes, producing four dimensions, as shown in Table 2. It is immediately apparent that the resultant components do not neatly reflect the totality of components of any of the present motivational theories.

\subsubsection{First Dimension: The Self-Determination Dimension}

Themes grouped under the self-determination dimension - a term borrowed from Deci \& Rayan (1980) - were themes 3 and 1, achieving positive outcomes (high grades). The achievement of this goal gives students feelings of satisfaction and happiness - this is the immediate reward (a positive enforcer in Skinner's terminology). Students obviously favour the recurrence of such a state of happy feelings, and so they know what to do - they, themselves, expect to have to work hard (expectancy theory). High grades are the instrument by which to get into the specialty they cherish; this is the valence element of the Valence, Instrumentality and Expectancy (VIE) theory. This dimension thus entails the goals, hopes and aspirations to be achieved from a university education (Locke \& Latham, 1984). This can be viewed as on the highest order of Maslow's motivation hierarchy, self-actualisation, or what Alderfer termed "the growth need".

Also included is theme H9, pleasing one's parents - a clear indication of the importance of family in Middle Eastern cultures. The family is a very important element of one's social life, and one that exerts a huge influence on the behaviour of the individual student in general. Students' desire to satisfy their parents and please them seems to be paramount. What McClelland termed as the need for affiliation, i.e. the desire to be accepted or liked by others (parents included), is self-evident in Middle Eastern families.

A further item, H10 (which is a personality trait), is included in this cluster of themes - the need to achieve merit, excellence and distinction. Here, students invest considerable energy, with perhaps little sleep, for the sake of attaining the learning target. McClelland termed one of his motivational theory components the "need to achieve." which is a compelling force driving the individual to succeed and get the job done. This can be seen as an expression of a type A personality trait.

\subsubsection{Second Dimension: Positive Academic Attitudes}

The second cluster, encompassing positive academic attitudes, relates to different aspects of university life itself. Themes in this cluster included item HI2, having positive attitudes towards university lecturers' style in lecturing and the way they (lecturers) relay facts to, and communicate in general with the students. This may be seen as a kind of positive relationship with a significant person or persons in the university, which is related to the need for social affiliation.

A further theme, $\mathrm{H} 5$, reflects students' love of or positive feelings and attitudes towards their speciality and the course materials, and $\mathrm{H} 6$ reflects the positive attitudes and feelings the students have for science and knowledge in general, which constitutes the heart and soul of university life and activity, producing knowledge and communicating it to others. Learning per se gives students feelings of satisfaction. 
The afore-mentioned traits correlate with the theme indicating students' love of challenging tasks, HI 3 . Whenever the learning task presents some difficulty, students double their efforts to understand and master it.

This may be due to having positive attitudes towards knowledge in general, and a positive preference for their speciality. Students with such attitudes tend to enjoy challenging tasks, since the expected reward can be the mere practising of the tasks they like best (Deci \& Rayan, 1992; Malone \& Lepper 1987).

\subsubsection{Third Dimension: Social Respect}

The third cluster of themes related mainly to item H8, the wish to have the respect and admiration of people in general, since "social values do not have a place for lazy people or laziness." The individual wants to have a respected position in society (social distinction). This includes item H9, pleasing one's parents, as an important aspect of having the respect and admiration of society, since those who have problematic relations with parents will be looked down upon by society at large. The cultural values of the society emphasise the place of parents in the family and society - "obeying parents." "showing respect for them." and "pleases them" is an integral part of the culture. Again, this social respect aspect is reco) in society recognised by the major theories of motivation: Maslow's social need to be accepted and loved by others, or at least to be accepted as a friend, also termed "relatedness" by Alderfer, and the "need for affiliation." the need to be liked and accepted by others and strive for friendship, by McClelland.

Society in general admires and values success and successful people who meet their targets in the shortest possible period of time. This cluster therefore includes item $\mathrm{H} 7$, the wish to graduate from university as quickly as possible. Those students who succeed in getting a job earlier than their peers are liked and admired by others, such as family and society at large. Early graduation and being liked by parents and society at large can be achieved if the students exhibit the need for achievement trait H10, which is the very strong desire to achieve merit, excellence and distinction.

\subsubsection{Fourth Dimension: Self-Monitoring}

The fourth cluster of themes included just two themes, the first of which is fear of failure, HI4, very frequently mentioned by students, and the second of which, H4, is achieving one's hopes and ambitions from a university education. It appears that this cluster is a direct reflection of goal-setting (Locke \& Latham, 1984; Drucker, 1954; Lewin, 1935), which pinpoints the importance of having specific performance goals to aim for. Such goals help the individual (worker or student) to evaluate their own performance and assess their progress. This also entails assessing what remains to be done to achieve one's ambitions or aspirations. Therefore the individual student needs to manage his/her own performance appropriately, and the fact that this cluster of themes is identified as a positive factor in students' motivation is $m$ line with the findings of Walters (1998) and Tang (1990), who stated that university students regulate their own level of motivation (effort) to complete their academic tasks, whenever they are challenged by difficult learning objectives.

Similarly, the fact that students assess their own study performance and the extent to which they regulate their time and effort in order to achieve their own goals mirrors valence theory, which views workers (students in the present case) as rational beings who compare costs and benefits. Students have certain cherished ambitions, i.e. goals highly desired by them. They therefore need to monitor their own progress towards those cherished goals in order to realise their own self-determination.

\subsection{University Students' Low Motivation Dimensions (Demotivators)}

A brief comparison of the frequencies and percentages of mention of motivating (encouraging) themes in Table 2 with the frequencies and percentages of mention of the demotivating (discouraging) themes reveals that the latter are considerably less in theme count and frequency of mention than the former. A possible explanation for this could well be the Freudian explanation, that individuals tend to suppress bad and painful incidents (experiences), while they tend to remember, talk about and perhaps elaborate upon happy situations (experiences).

Employing Principal Component Analysis to categorise the study-discouraging themes into clusters resulted in just three groupings, as follows:

\subsubsection{First Dimension: Negative Personal Feelings and Circumstances}

This cluster of themes includes items L3, which incorporates negative and unsuitable personal circumstances such as illness, having to do another job, thinking of the future, having an interest in football matches; and L2, which is being in a transition state - wanting to go to another university, another department, or another country. Students clearly cannot concentrate whole-heartedly on learning tasks if they are paying considerable attention to other actual or pending events. 
A further theme in this cluster is LI, feelings of frustration, boredom, and hopelessness. With such negative feelings and unhelpful personal states, students cannot feel inspired with the required motivation. Added to this cluster is theme L4, which is the feeling that the learning tasks are very difficult, they cannot understand them and therefore they cannot master them.

The final theme grouped in this cluster is L8, incorporating negative family circumstances such as tension among family members, poor parental health, helping out other family members and numerous social commitments. The strong family ties evident in Jordan's culture mean that any unfortunate conditions will certainly affect everybody within the family, including the student member.

\subsubsection{Second Dimension: Learned Helplessness}

The second cluster of themes pertains to the relation between students and their specialties. Theme L6 reveals that some students actually hate their specialty. In many cases, this situation results from the procedure universities in Jordan follow in admitting students to their different colleges and departments. A difference of a fraction of a percentage point in the school-leaving ("Tawjihi") examinations can place a student in a specialty that they do not like. Similarly, theme L10 reveals that some students cannot adjust to university life in general. They go to university with certain wrong impressions and beliefs about university life and learning regulations. They therefore need guidance and counselling. Watkins \& Hattie (1981) found that university students who are in dire need of counselling are invariably first-year students. Unfortunately universities in Jordan in general offer very little orientation or counseling to students.

The third item in this cluster of themes relates to unfavourable family circumstances. As previously mentioned, the family's influence is paramount. Parents may advise their sons and daughters to go into specialities they may dislike, and family circumstances may hinder students academic performance. The outcome of such unhelpful family situations could be a very negative learning experience and performance, resulting in low grades. Negative feedback often creates feelings of frustration, and perhaps also helplessness. If a student's previous performance repeatedly brought negative outcomes, then he /she will stop trying to achieve (Skinner's theory states that behaviour is the function of the behavior consequences). The individual student stops trying to learn (L9). Other students may learn by observation and stop trying also.

\subsubsection{Third Dimension: Negative Self-Monitoring}

The third and final cluster involves just two themes: the first theme, L7, indicates that whenever the learning task is very easy, students allocate to that learning task very little time and effort; the second theme, L5, reveals that students may have wrong information and beliefs about university regulations and standards. One such belief could be that mastering learning tasks at university does not require hard work for sustained periods. Watkins \& Hattie's (1981) results indicate that first-year students in other cultures may also have wrong ideas and beliefs about the university learning system. Cognitive counselling is therefore needed to help students acquire the necessary correct information about the university system of learning, since this is an essential aspect of students' successful self-evaluation and regulation of the effort needed to achieve their goals. Certainly a negative state of self-monitoring (self-management) such as this will clearly have negative outcomes.

\section{Conclusion}

Employing any of the currently available learning motivation theories and questionnaires to study the academic motivation of university students in Jordan could not possibly have given the results detailed above, even though such methodology may have, in the end, been seen to validate the employed theory or questionnaire. This finding alone must be of value to cross-cultural studies and the theoretical formulations of motivational theories.

Among the currently available motivational theories (need, rational, process, learning, self-determination, etc.) each has certain components which are very similar to one or more of the motivational themes identified in the present investigation. This is an indication, then, that none of the present theories can on its own explain the university learning situation, which is very complex indeed.

The learning motivation of the subjects studied appears to be affected by seven major motivational dimensions. The first four dimensions have a positive effect on university students' state of motivation and this high motivation state is found to be caused simultaneously by various types of theme - intrinsic, extrinsic, social, and reward. The remaining three dimensions have a negative effect on the students' motivational state and cause the emergence of a state of low motivation.

The present study reveals that university students' high motivation state depends - in the eyes of the students themselves - on the presence of certain motivational themes, and the absence of certain demotivational themes. This study therefore has an important message to pass to the university change planners in their endeavours to 
continuously improve the quality standards of university graduates: they need to focus their attention on the university students themselves and strive to enable the students to experience more of those positive motivating factors and less of the negative demotivating factors. This does not, of course, mean abandoning attention to other aspects of the learning situation, such as the classroom, learning system and learning situation leader, i.e. the instructor or lecturer.

Certain limitations of this study have, however, to be addressed before applying the present study results to real situations. The subjects investigated were all following a university elective course. There is therefore a need to investigate students studying non-elective courses in different university colleges and departments before generalising the results to other students. In addition, the fact that this study is exploratory in nature requires that further studies should be conducted with stringent designs (predictive design, for example) in order to validate the different motivational components, and relate these to certain aspects of learning activity such as high or low task mastery and year of study in the university. Other studies could also be of theoretical and practical importance, such as the interaction between the previously-mentioned themes and aspects of personality, self-esteem, and locus of control, for example.

\section{Policy Implications}

The results of this study show that the state of university students' high academic motivation is associated with activities that students like and are interested in practicing. Those activities also are accompanied by happy feelings and states of pleasure. Hence, students will dedicate more time and effort to perform the needed academic activities such as participation in classroom work and attention concentration. The expected results are better understanding and better ability to recall what has been learned.

On the other hand, the study results show that university students low state of academic motivation is associated with activities accompanied by unhappy feelings and states of pain. Since university students are keen to avoid painful feelings, they most probably will dedicate very little time and effort to performing such academic activities.

The above mentioned conclusions show that policies practiced by Jordan's government managed universities should take into consideration students career and vocational preferences. Placing the individual student in a certain department should take into account his/her vocational interests. Therefore, the general education authorities at the secondary education level should establish centers that provide secondary level students with advice concerning their future job options. Proper career and vocational guidance based on matching students' aptitudes, values, and personality characteristics is valid and successful guidance. Such centers well help pupils make mature career choices based on proper vocational guidance and advice which relies upon psychological techniques and tests.

Developing such centers and taking into account students career interests will provide university classrooms with students who are keen to participate in university academic activities and whose attention concentration and understanding most probably will be far better.

It is expected that university students' academic achievement standards will be far better if the above mentioned recommendation is taken seriously and implemented in the university. Nevertheless, this will be only a partial remedy, since university students' academic achievement is a complex phenomenon and is affected by several factors, such as the physical classroom, university managerial system and staff, academic staff, social values and system beyond the university's physical borders.

\section{References}

Adams, J. S. (1965). Inequity in Social Exchange. In L. Berkowitz (Ed.), Advances in Experimental Social Psychology (vol.2). New York: Academic Press.

Alderfer, C. P. (1972). Existence, Relatedness and Growth: Human Needs in Organizational Settings. New York: Free Press.

Biggs, J. (1987). Study Process Questionnaire Manual. Melbourne: Australian Council for Educational Research.

Biggs, J. B. (1978). Individual and Group Differences in Study Processes. Br. J. educ. Psychol., 48, 266-279. http://dx.doi.org/10.1111/j.2044-8279.1978.tb03013.x

David, C. C. (1997). Doing Quantitative Psychological Research: From Design to Report. UK: Psychology Press.

Deci, E. L, Vallerand, J., Pelletier, L. G., \& Ryan, R. M. (1991). Motivation and Education: The Self-determination Perspective. Educational Psychologyist, 26, 325-346. 
Entwistle, N. J., \& Entwistle, A. (1991). Contrasting froms of Understanding for Degree Examinations; The Student Experience and its Implications. Higher Eucation, 22, 205-227. http://dx.doi.org/10.1007/BF00132288

Entwistle, N. J., Nisbet, J., Entwistle, D., \& Cowell, M. D. (1971). The Academic Performance of Students: Prediction from Scales of Motivation and Study Methods. Br. J. educ. Psychol., 41, 258-267. http://dx.doi.org/10.1111/j.2044-8279.1971.tb00670.x

Flanagan, J. C. (1954). The Critical Incident Technique. Psychological Bulletin, 51, 327-335. http://dx.doi.org/10.1037/h0061470

Glynis, M. B., Hammond, S., \& Fife-Schaw, C. (2000). Research Methods in Psychology (2nd ed.). London: SAGE Publications.

Herzberg, F., Mausner, B., \& Snyderman, B. B. (1959). The Motivation to Work (2nd ed.). New York: Wiley.

Hiroto, D. S. (1974). Laws of Control and Learned Helplessness. Journal of Experimental Psychology, 102, 187-193. http://dx.doi.org/10.1037/h0035910

Hwang, Y. S. (2000). Asian American College Student's Motivation in Education. Paper Presented at the Annual Meeting of the American Education Research Association, New Orleans.

Hwang, Y. S., Echols, C., \& Verongistinos, K. (2002). Multidimensional Academic Motivation of High Achieving African American Students.

Jacobs, P. A., \& Newstead, S. E. (2000). The Nature and Development of Student Motivation. Br. J. of Educ. Psychol., 70.

Lavery, L. (1999). Ethnic Group Differences in the Academic Motivation of University Students.

Likert, R. (1967). Human Organization. New York: McGraw-Hill.

Locke, E. A., \& Latham, G. P. (1984). Goal Seting: A Motivational Technique that Works. Englewood Cliffs, NJ: Prentice-Hall.

Maslow, A. H. (1943). A Theory of Human Motivation. Psychological Review, 50, 370-396. http://dx.doi.org/10.1037/h0054346

McClelland, D. C. (1985). Human Motivation. Glenview, IL: Scott Foresman.

Radford, J., \& Holdstock, L. (1993). What Students Want: Objectives of First Year Psychology Students in Ireland, Norway, Portugal, Spain and the United Kingdom. Psychology Teaching Review, 2, 39-49.

Robert, S., \& Sommer, B. B. (1986). A Practical Guide to Behavioral Research tools and Techniques (2nd ed.). Oxford, New York: Oxford University Press.

Seiigman, M. E. P., \& Maier, S. F. (1967). Failure to Escape. Journal of Experimental Psychology, 74, 1-9. http://dx.doi.org/10.1037/h0024514

Tang, T. L. (1990). Factors Affecting Intrinsic Motivation Among University Students in Taiwan. The Journal of Social Psychology, 13(2), 219-230. http://dx.doi.org/10.1080/00224545.1990.9924572

Tannenbaum, A. S. (1980). Organizational Psychology. In H. C. Triandis and R. W. Brislin (Eds.), Handbook of Cross-cultural Psychology, 5. Boston: Allyn and Bacon.

Van Etten, S., Pressley, M., Freebern, G., \& Eschevarria, M. (1998). An Interview Study of College Freshmen's Beliefs about their Academic Motivation. European Journal of Education, 13(1), 105-243.

Vroom, V. H. (1964). Work and Motivation. New York: Wiley.

Watkins, D., \& Hattie, J. (1981). The Learning Processes of Australian University Students: Investigations of Contextual and Personological Factors. Br. J. educ. Psychol., 51, 384-393. http://dx.doi.org/10.1111/j.2044-8279.1981.tb02494.x

Wolters, C. A. (1998). Self-Regulated Learning and College Students' Regulation of Motivation. Journal of Educational Psychology, 90(2), 224-235. http://dx.doi.org/10.1037/0022-0663.90.2.224 